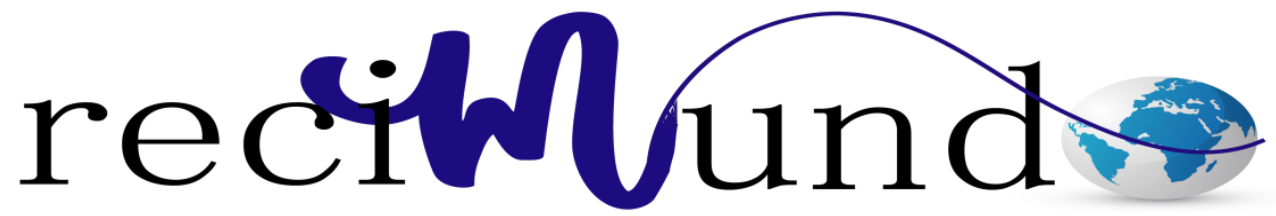

Revista Científica Mundo de la Investigación y el Conocimiento

Md. Geovanna María Corral Sánchez a; Md. Luisana Belén Solórzano López ${ }^{\text {; }}$ Md. Karina Lisseth Mendoza Hidalgo ${ }^{\text {c }}$, Md. Diana Karen Vélez Mera ${ }^{\text {d }}$

Síndrome metabólico y trastornos nutricionales: Un problema de salud pública

Revista Científica Mundo de la Investigación y el Conocimiento. Vol. 2 núm.2, mayo, ISSN: 2588-073X, 2018, pp. 631-639

DOI: 10.26820/recimundo/2.(2).2018.631-639

Editorial Saberes del Conocimiento

Recibido: 05/12/2017

Aceptado: 15/02/2018

a. Hospital General de Chone Napoleón Dávila Córdova; geovanna.corral@ hospitalgeneralchone.gob.ec

b. Hospital General de Chone Napoleón Dávila Córdova; luisana.solorzano@ hospitalgeneralchone.gob.ec

c. Hospital General de Chone Napoleón Dávila Córdova; karina.mendoza@ hospitalgeneralchone.gob.ec

d. Hospital General de Chone Napoleón Dávila Córdova; diana.velez@ hospitalgeneralchone.gob.ec 


\section{Síndrome metabólico y trastornos nutricionales: Un problema de salud}

pública

Vol. 2, núm. 2., (2018)

Md. Geovanna María Corral Sánchez; Md. Luisana Belén Solórzano López; Md. Karina Lisseth Mendoza Hidalgo; Md. Diana Karen Vélez Mera

\section{RESUMEN}

En el presente trabajo de investigación se abordaron las consecuencias inherentes síndrome metabólico (SM) y trastornos nutricionales, enfocándolo como un problema de salud pública. La causa de este padecimiento está muy ligado a los estilos de vida de cada persona, como la sobrealimentación y la inactividad física, además de factores genéticos. Este síndrome conlleva una serie de factores de riesgo como: obesidad central, dislipidemias, anomalías en el metabolismo de la glucosa e hipertensión arterial. Las estadísticas reflejan que las personas con SM, que representan un 20 a 25\% (aproximadamente) de la población mundial, tienen tres veces mayores probabilidades de sufrir un ataque cardíaco o un accidente cerebro vascular y dos veces más de morir por estas causas, que las personas que no lo padecen. Se pudo determinar que el ejercicio físico regular es, junto con la alimentación adecuada, la otra medida no farmacológica esencial para la prevención de la DM y SM. La actividad física ayuda en la mejora del control glucémico, la sensibilidad a la insulina y la reducción de las complicaciones. Se recomienda que las personas con sobrepeso u obesas; además de las que presenten factores de riesgo como diabetes o enfermedades cardiovasculares se realicen la evaluación del síndrome metabólico.

Palabras clave: Síndrome metabólico, obesidad, nutrición, dieta, estilos de vida, salud, factores de riesgo. 


\title{
Síndrome metabólico y trastornos nutricionales: Un problema de salud \\ pública
}

Vol. 2, núm. 2., (2018)

Md. Geovanna María Corral Sánchez; Md. Luisana Belén Solórzano López; Md. Karina Lisseth

Mendoza Hidalgo; Md. Diana Karen Vélez Mera

\begin{abstract}
In the present research work, the inherent consequences of the metabolic syndrome (MS) and nutritional disorders are addressed, focusing it as a public health problem. The cause of this condition is closely linked to the lifestyles of each person, such as overfeeding and physical inactivity, in addition to genetic factors. This syndrome involves a series of risk factors such as: central obesity, dyslipidemias, abnormalities in glucose metabolism and arterial hypertension. The statistics that people with MS, which account for 20 to $25 \%$ of the world's population, are three times more likely to suffer a heart attack or stroke and twice as likely to die from these causes, than people who do not. They suffer It was determined that physical exercise is regular, along with adequate nutrition, the other non-pharmacological measure essential for the prevention of DM and MS. Physical activity helps in the improvement of glycemic control, insulin sensitivity and reduction of complications. It is recommend that people who are overweight or obese; In addition to those that present risk factors such as diabetes or cardiovascular diseases, the evaluation of the metabolic syndrome is carried out.
\end{abstract}

Key words: Metabolic syndrome, obesity, nutrition, diet, lifestyles, health, risk factors 


\section{Síndrome metabólico y trastornos nutricionales: Un problema de salud pública}

Vol. 2, núm. 2., (2018)

Md. Geovanna María Corral Sánchez; Md. Luisana Belén Solórzano López; Md. Karina Lisseth Mendoza Hidalgo; Md. Diana Karen Vélez Mera

\section{Introducción.}

El síndrome metabólico (SM) comprende un conjunto de factores de riesgo representados por obesidad central, dislipidemias, anomalías en el metabolismo de la glucosa e hipertensión arterial (HTA), asociados a resistencia a la insulina (RI), que incrementa el riesgo de enfermedad cardiovascular y de diabetes mellitus (Soca, y otros, 2012)

La causa de estos problemas está dada por la combinación de factores genéticos y socio ambientales relacionados a los cambios en los estilos de vida, especialmente la sobrealimentación y la inactividad física. Sin embargo, hay que considerar que algunos individuos están genéticamente predispuestos a padecerla (Pajuelo \& Sánchez, El síndrome metabólico en adultos, en el Perú, 2007).

La obesidad y el sedentarismo son factores de riesgo subyacentes en la ruta patogénica de este síndrome, por tanto, la modificación de los hábitos de vida es una intervención de primera línea en la prevención y tratamiento de la resistencia insulínica, la hiperglucemia, la dislipemia aterogénica y la hipertensión arterial. (Matía, Lecumberri, \& Calle, 2007)

El síndrome metabólico está asociado fuertemente a la expansión de la epidemia mundial de diabetes tipo 2 y de enfermedades cardiovasculares, según datos recientes de la Federación Internacional de Diabetes (FID). Las personas con síndrome metabólico aproximadamente 20 a $25 \%$ de la población mundial tienen una probabilidad tres veces mayor de sufrir un ataque cardíaco o un accidente cerebro vascular y dos veces más de morir por estas causas, que las 


\section{Síndrome metabólico y trastornos nutricionales: Un problema de salud \\ pública}

Vol. 2, núm. 2., (2018)

Md. Geovanna María Corral Sánchez; Md. Luisana Belén Solórzano López; Md. Karina Lisseth Mendoza Hidalgo; Md. Diana Karen Vélez Mera

personas que no lo padecen (Pajuelo \& Sánchez, El síndrome metabólico en adultos, en el Perú, 2007).

Asimismo, el incremento en la prevalencia de este conjunto de alteraciones a nivel mundial es alarmante, más aún si se toma en cuenta que es considerado el mejor predictor del desarrollo de diabetes, que solo la intolerancia a la glucosa. El impacto del SM ha sido demostrado por el incremento de la enfermedad ateroesclerótica subclínica en pacientes con el síndrome, aún sin el diagnóstico de diabetes (Lizarzaburu, 2013).

La atención del SM comprende dos objetivos, la reducción de las causas subyacentes: obesidad e inactividad física y el tratamiento de los factores de riesgo. Una modificación de los estilos de vida y, en especial, de los hábitos nutricionales y de la actividad física, es la piedra angular de su manejo. La disminución de peso y el incremento de la actividad física conducen a la reducción de los factores de riesgo al mejorar la sensibilidad a la insulina (Soca, y otros, 2009).

\section{Materiales y métodos.}

La investigación científica sirve para la producción de conocimientos y la interpretación de una realidad. En el presente trabajo se explica el hecho mediante la investigación documental que consiste en un proceso sistemático de indagación, recolección, organización, análisis e interpretación de información o datos en torno a un determinado tema. Al igual que otros tipos de investigación, éste es conducente a la construcción de conocimientos. (Alfonzo, 1994) 


\section{Síndrome metabólico y trastornos nutricionales: Un problema de salud}

pública

Vol. 2, núm. 2., (2018)

Md. Geovanna María Corral Sánchez; Md. Luisana Belén Solórzano López; Md. Karina Lisseth Mendoza Hidalgo; Md. Diana Karen Vélez Mera

La investigación documental tiene la particularidad de utilizar como una fuente primaria de insumos, más no la única y exclusiva, el documento escrito en sus diferentes formas: documentos impresos, electrónicos y audiovisuales. Las fuentes impresas incluyen: libros enciclopedias, revistas, periódicos, diccionarios, monografías, tesis y otros documentos. Asimismo, se pueden usar fuentes electrónicas como: correos electrónicos, CD roms, base de datos, revistas y periódicos en línea y páginas web. (Oscar, 2003)

Para la elaboración de este estudio se recopiló información y datos de diversos artículos médicos para abordar de forma correcta el síndrome metabólico y los trastornos nutricionales. Siempre privilegiando los más actuales y los que contuvieran más datos duros, para poder hacer interpretación de la realidad lo más acorde a los tiempos modernos.

\section{Resultados.}

En cuanto a cambios en la alimentación que ayuden a prevenir la aparición de DM, se debe mantener el índice de masa corporal (IMC) por debajo de $25 \mathrm{Kg} / \mathrm{m}^{2}$, realizando algunas modificaciones nutricionales como son: dieta rica en fibra y grasas poli-insaturadas con respecto a las grasas saturadas (aumento del ratio polinsaturadas/saturadas), disminuir la ingesta de grasas trans procedentes de la hidrogenación de los ácidos grasos, y limitar la ingesta de alimentos con alto IG. (Matía, Lecumberri, \& Calle, 2007)

Dada la estrecha relación entre obesidad abdominal e insulino resistencia se planteó que la obesidad abdominal sería el más importante de los factores de riesgo y el que conlleva al desencadenamiento de las demás anormalidades en el síndrome, ya que implica el aumento y 


\section{Síndrome metabólico y trastornos nutricionales: Un problema de salud \\ pública}

Vol. 2, núm. 2., (2018)

Md. Geovanna María Corral Sánchez; Md. Luisana Belén Solórzano López; Md. Karina Lisseth Mendoza Hidalgo; Md. Diana Karen Vélez Mera

acúmulo de grasa a nivel visceral (depósito de tejido graso principalmente en hígado, músculo y páncreas), tendría la mayor implicancia en el desarrollo del síndrome. Esta grasa visceral implica la formación en el tejido graso de sustancias químicas llamadas adipoquinas, que favorecen estados proinflamatorios y protrombóticos, que a su vez van a conducir o contribuir al desarrollo de insulino resistencia, hiperinsulinemia, alteración en la fibrinólisis y disfunción endotelial (Lizarzaburu, 2013).

El ejercicio físico regular es, junto con la alimentación adecuada, la otra medida no farmacológica esencial para la prevención de la DM y SM Según la ADA, la actividad física tiene un nivel de evidencia B en la prevención de la DM y en la mejora del control glucémico, la sensibilidad a la insulina y la reducción de las complicaciones. (Matía, Lecumberri, \& Calle, 2007)

\section{Conclusiones.}

De igual manera con respecto a la alimentación, diversos estudios en los que se sigue un patrón de dieta mediterránea han demostrado disminuir la incidencia de DM y por lo tanto de SM. Asimismo, se ha demostrado que a mayor adherencia a la dieta existe menor mortalidad global, así como por enfermedad cardiovascular y cáncer. La dieta mediterránea, definida como una dieta saludable, se caracteriza por un elevado consumo de verduras, legumbres, frutas, frutos secos, cereales integrales y aceite de oliva, bajo consumo de grasas saturadas, moderada-alta ingesta de pescado, moderado-bajo consumo de leche y queso, baja ingesta de carne roja y una moderada y regular ingesta de vino con las comidas. Por otra parte, hay evidencias de que el 


\section{Síndrome metabólico y trastornos nutricionales: Un problema de salud pública}

Vol. 2, núm. 2., (2018)

Md. Geovanna María Corral Sánchez; Md. Luisana Belén Solórzano López; Md. Karina Lisseth Mendoza Hidalgo; Md. Diana Karen Vélez Mera

elevado consumo de carne roja y leche entera está asociado a un aumento de la resistencia a la insulina, por tanto a DM y SM. (Matía, Lecumberri, \& Calle, 2007)

La circunferencia abdominal es el mejor indicador antropométrico de carácter predictivo de enfermedades cardiovasculares. Por esa razón, se lo considera como uno de los criterios más importantes para definir síndrome metabólico. En ese sentido, se puede inferir que los incrementos que se dan en función del tiempo van a repercutir en un aumento del síndrome metabólico. (Pajuelo, Bernui, Nolberto, Peña, \& Zevillanos, 2007).

\section{Recomendaciones}

La evaluación del síndrome metabólico debe realizarse en todas las personas con sobrepeso u obesas y en aquellos que presenten algún factor de riesgo de diabetes o enfermedad cardiovascular, como hipertensión, diabetes, dislipidemias o sedentarismo. (Lizarzaburu, 2013).

Asimismo, se deben tomar cartas en el asunto, dado que este tipo de expresiones de anormalidad tiene una tendencia epidemiológica a continuar incrementándose, acarreando problemas no solo para la salud sino para la economía del país. Otro hecho a tomar en cuenta, dentro de las características epidemiológicas del síndrome metabólico es que, conforme avanza la edad la prevalencia aumenta y como la expectativa de vida al nacer ha mejorado, se van a presentar más los problemas. (Pajuelo \& Sánchez, El síndrome metabólico en adultos, en el Perú, 2007).

Por otra parte se recomienda restringir la ingesta de grasa saturada $(<7 \%$ de las Kcal diarias), grasa trans y colesterol a favor de las grasas mono insaturadas (hasta el 20-25\% de las 


\section{Síndrome metabólico y trastornos nutricionales: Un problema de salud \\ pública}

Vol. 2, núm. 2., (2018)

Md. Geovanna María Corral Sánchez; Md. Luisana Belén Solórzano López; Md. Karina Lisseth Mendoza Hidalgo; Md. Diana Karen Vélez Mera

Kcal diarias) y poli insaturadas, y limitar el sodio y los azúcares refinados. El consumo de frutas, verduras y cereales integrales debe ser elevado, potenciando también la ingesta de pescado. Incrementar la actividad física habitual es un componente fundamental recomendable del cambio en el estilo de vida (Matía, Lecumberri, \& Calle, 2007)

\section{Bibliografía.}

Alfonzo, I. (1994). Técnicas de investigación bibliográfica. . Caracas: Contexto Ediciones.

Lizarzaburu, J. C. (2013). Síndrome metabólico: concepto y aplicación práctica. An. Fac. med. vol.74 no.4.

Matía, P., Lecumberri, E., \& Calle, A. (2007). Nutrición y síndrome metabólico. Rev. Esp. Salud Publica vol.81 no.5, 15-35.

Oscar, M. (2003). Fundamentos de la Investigación Documental y la. Manual para la elaboración y presentación de la monografía, 20.

Pajuelo, J., \& Sánchez, J. (2007). El síndrome metabólico en adultos, en el Perú. An. Fac. med. v. 68 n.1, 5-20.

Pajuelo, J., Bernui, I., Nolberto, V., Peña, A., \& Zevillanos, L. (2007). Síndrome metabólico en adolescentes con sobrepeso y obesidad. An. Fac. med. v.68 n.2.

Soca, P., Cruz, W., González, J., Cardona, X., Cruz, L., \& Hernández, M. (2009). Efectos beneficiosos de cambios en la dieta y ejercicios físicos en mujeres obesas con síndrome metabólico. Panorama Cuba y salud, 29-36.

Soca, P., Peña, I., Niño, S., Cruz, W., Niño, A., \& Ponce, D. (2012). Ensayo clínico aleatorio: papel de la dieta y ejercicios físicos en mujeres con síndrome metabólico. Atencion Primaria, Elsevier, 383-393. 